\title{
NULL DISTRIBUTION OF MULTIPLE CORRELATION COEFFICIENT UNDER MIXTURE NORMAL MODEL
}

\author{
HYDAR ALI and DAYA K. NAGAR
}

Received 14 April 2001

\begin{abstract}
The multiple correlation coefficient is used in a large variety of statistical tests and regression problems. In this article, we derive the null distribution of the square of the sample multiple correlation coefficient, $R^{2}$, when a sample is drawn from a mixture of two multivariate Gaussian populations. The moments of $1-R^{2}$ and inverse Mellin transform have been used to derive the density of $R^{2}$.
\end{abstract}

2000 Mathematics Subject Classification: 62H10, 62H15.

1. Introduction. Suppose that $\mathbf{x}(p \times 1), \boldsymbol{\mu}(p \times 1)$, and $\Sigma(p \times p)>0$ are partitioned as $\mathbf{X}=\left(\begin{array}{c}x_{1} \\ \mathbf{x}^{(2)}\end{array}\right), \boldsymbol{\mu}=\left(\begin{array}{c}\mu_{1} \\ \boldsymbol{\mu}^{(2)}\end{array}\right)$, and $\Sigma=\left(\begin{array}{cc}\sigma_{11} & \boldsymbol{\sigma}_{21}^{\prime} \\ \boldsymbol{\sigma}_{21} & \Sigma_{22}\end{array}\right)$, where $\mathbf{x}^{(2)}=\left(x_{2}, \ldots, x_{p}\right)^{\prime}$ and $\boldsymbol{\mu}^{(2)}=\left(\mu_{2}, \ldots, \mu_{p}\right)^{\prime}$ are $(p-1) \times 1$ and $\Sigma_{22}$ is $(p-1) \times(p-1)$, so that $\operatorname{Var}\left(x_{1}\right)=\sigma_{11}, \operatorname{Cov}\left(\mathbf{x}^{(2)}\right)=\Sigma_{22}$, and $\boldsymbol{\sigma}_{12}$ is the $(p-1) \times 1$ vector of covariances between $x_{1}$ and $x_{2}, \ldots, x_{p}$. The multiple correlation coefficient between $x_{1}$ and $\mathbf{x}^{(2)}$, denoted by $\bar{R}_{1 \cdot 2 \ldots p}$, is defined as

$$
\bar{R}_{1 \cdot 2 \cdots p}=\left(\frac{\boldsymbol{\sigma}_{21}^{\prime} \Sigma_{22}^{-1} \boldsymbol{\sigma}_{21}}{\sigma_{11}}\right)^{1 / 2} .
$$

Let $A$ be the sample sum of squares and products matrix formed from $N$ independent observations on $\mathbf{x}$. Partition $A$ as $A=\left(\begin{array}{ll}a_{11} & \mathbf{a}_{21}^{\prime} \\ \mathbf{a}_{21} & A_{22}\end{array}\right)$, where $A_{22}$ is $(p-1) \times(p-1)$. The sample multiple correlation coefficient between $x_{1}$ and $\mathbf{x}^{(2)}$ is defined by

$$
R=\left(\frac{\mathbf{a}_{21}^{\prime} A_{22}^{-1} \mathbf{a}_{21}}{a_{11}}\right)^{1 / 2} .
$$

It is well known that, when the underlying population is normal, the random matrix $A$ has Wishart distribution with $n=N-1$ degrees of freedom and parameter matrix $\Sigma$. Further, $\bar{R}_{1 \cdot 2 \ldots p}=0$ if and only if $x_{1}$ is independent of $\mathbf{x}^{(2)}=\left(x_{2}, \ldots, x_{p}\right)$. Furthermore, when the population multiple correlation coefficient $\bar{R}_{1 \cdot 2 \ldots p}$ is zero, the distribution of $R^{2}$ is beta with parameters $(1 / 2)(p-1)$ and $(1 / 2)(N-p)$.

In practice, it is often the case that the random variables are not normally distributed. When such is the case, how would the departure from the normality affect the conventional inference procedure? Specifically, one may wonder what would be the sampling distributions of some commonly used statistics? For providing some answers to the above questions, Srivastava and Awan [9] and Tan [11] derived the distribution of the sample sum of squares and products matrix when sampling from a mixture of two multivariate normal distributions. The normal mixture is defined as follows:

$$
f(\mathbf{x})=\epsilon N_{p}\left(\boldsymbol{\mu}_{1}, \Sigma ; \mathbf{x}\right)+(1-\epsilon) N_{p}\left(\boldsymbol{\mu}_{2}, \Sigma ; \mathbf{x}\right), \quad \mathbf{x} \in \mathbb{R}^{p},
$$


where

$$
\begin{aligned}
& N_{p}(\boldsymbol{\mu}, \Sigma ; \mathbf{x}) \\
& \quad=(2 \pi)^{-(1 / 2) p} \operatorname{det}(\Sigma)^{-1 / 2} \exp \left\{-\frac{1}{2}(\mathbf{x}-\boldsymbol{\mu})^{\prime} \Sigma^{-1}(\mathbf{x}-\boldsymbol{\mu})\right\}, \quad \mathbf{x} \in \mathbb{R}^{p}, \boldsymbol{\mu} \in \mathbb{R}^{p}, \Sigma>0,
\end{aligned}
$$

and $1-\epsilon$ is known as the degree of contamination. This model is very common in medical, biological, and agricultural experiments (Titterington et al. [12]). For results on the distribution theory and robustness studies of certain test statistics when sampling from a mixture normal model, see Srivastava [8], Srivastava and Awan [9, 10], Kabe and Gupta [5], Amey and Gupta [2], and Nagar and Castañeda [7].

Srivastava [8], using certain transformations, derived the null distribution of multiple correlation coefficient when sampling from a mixture of two multivariate normal distributions (see also Gupta and Kabe [3]). Amey [1] integrated the joint density of $a_{11}, \mathbf{a}_{21}$, and $A_{22}$ suitably to derive the density of $R^{2}$ and studied its robustness.

In this article, we derive the null distribution of $R^{2}$ when sampling from a mixture of two multivariate normal distributions. First, we derive the $h$ th null moment of $1-R^{2}$. Then, by using the inverse Mellin transform, the density of $1-R^{2}$ is obtained from which the density of $R^{2}$ is deduced.

Note that $R^{2}$ is a function of the elements of sample sum of squares and products matrix $A$. Therefore, in our derivation, we use the distribution of $A$ when sampling from the above model. Srivastava and Awan [9] and Tan [11] have shown that the density of $A$, when sampling from (1.3), is a binomial sum of linear noncentral Wishart densities:

$$
f(A)=\sum_{\gamma=0}^{N}\left(\begin{array}{l}
N \\
\gamma
\end{array}\right) \epsilon^{\gamma}(1-\epsilon)^{N-\gamma} W_{p}\left(n, \Sigma, c_{\gamma}^{2} \Sigma^{-1} \boldsymbol{v} \boldsymbol{v}^{\prime} ; A\right),
$$

where $n=N-1, c_{\gamma}^{2}=\gamma(N-\gamma) / N$, and $\boldsymbol{v}=\left(\boldsymbol{\mu}_{1}-\boldsymbol{\mu}_{2}\right)$. Here $W_{p}\left(n, \Sigma, c_{\gamma}^{2} \Sigma^{-1} \boldsymbol{v} v^{\prime} ; A\right)$ represents the noncentral Wishart density with $n$ degrees of freedom and noncentrality parameter matrix $c_{\gamma}^{2} \Sigma^{-1} \boldsymbol{v} \boldsymbol{v}^{\prime}$ defined by

$$
K_{p}(n, \Sigma, \boldsymbol{v}) \operatorname{etr}\left(-\frac{1}{2} \Sigma^{-1} A\right) \operatorname{det}(A)^{(1 / 2)(n-p-1)}{ }_{0} F_{1}^{(p)}\left(\frac{1}{2} n ; \frac{1}{4} c_{\gamma}^{2} \Sigma^{-1} A \Sigma^{-1} \boldsymbol{v} \boldsymbol{v}^{\prime}\right),
$$

where

$$
K_{p}(n, \Sigma, \boldsymbol{v})=\left\{2^{(1 / 2) p n} \Gamma_{p}\left(\frac{1}{2} n\right) \operatorname{det}(\Sigma)^{(1 / 2) n}\right\}^{-1} \operatorname{etr}\left(-\frac{1}{2} c_{\gamma}^{2} \Sigma^{-1} \boldsymbol{v} \boldsymbol{v}^{\prime}\right)
$$

and $\Gamma_{m}(a)=\pi^{(1 / 4) m(m-1)} \prod_{j=1}^{m} \Gamma(a-(1 / 2)(j-1))$.

2. Null moments of $1-R^{2}$. In this section, we derive moments of $1-R^{2}$ when $\bar{R}_{1 \cdot 2 \cdots p}=0$ (or equivalently $\boldsymbol{\sigma}_{21}=\mathbf{0}$ ). Let $\Sigma_{0}=\left(\begin{array}{cc}\sigma_{11} & 0^{\prime} \\ 0 & \Sigma_{22}\end{array}\right)$ and $U=1-R^{2}$. Since $a_{11}$ is scalar, then

$$
U=1-R^{2}=1-\frac{\mathbf{a}_{21}^{\prime} A_{22}^{-1} \mathbf{a}_{21}}{a_{11}}=\frac{\operatorname{det}(A)}{a_{11} \operatorname{det}\left(A_{22}\right)} .
$$


The $h$ th null moment of $U$ is given by

$$
\begin{gathered}
E\left(U^{h}\right)=\sum_{\gamma=0}^{N}\left(\begin{array}{c}
N \\
\gamma
\end{array}\right) \epsilon^{\gamma}(1-\epsilon)^{N-\gamma} E_{\gamma}\left(U^{h}\right), \\
E_{\gamma}\left(U^{h}\right)=K_{p}\left(n, \Sigma_{0}, \boldsymbol{v}\right) \int_{A>0} \operatorname{etr}\left(-\frac{1}{2} \Sigma_{0}^{-1} A\right) a_{11}^{-h} \operatorname{det}\left(A_{22}\right)^{-h} \\
\times \operatorname{det}(A)^{(1 / 2)(n-p-1)+h}{ }_{0} F_{1}^{(p)}\left(\frac{1}{2} n ; \frac{1}{4} c_{\gamma}^{2} \Sigma_{0}^{-1} A \Sigma_{0}^{-1} \boldsymbol{v} \boldsymbol{v}^{\prime}\right) d A .
\end{gathered}
$$

Replacing $a_{11}^{-h}$ and $\operatorname{det}\left(A_{22}\right)^{-h}$ by their integral representations, namely

$$
\begin{aligned}
a_{11}^{-h}= & \frac{1}{2^{h} \Gamma(h)} \int_{0}^{\infty} \exp \left(-\frac{1}{2} a_{11} y_{1}\right) y_{1}^{h-1} d y_{1}, \quad \operatorname{Re}(h)>0, \\
\operatorname{det}\left(A_{22}\right)^{-h}= & \frac{1}{2^{(p-1) h} \Gamma_{p-1}(h)} \int_{Y_{22}>0} \operatorname{etr}\left(-\frac{1}{2} A_{22} Y_{22}\right) \\
& \times \operatorname{det}\left(Y_{22}\right)^{h-(1 / 2)(p-1+1)} d Y_{22}, \quad \operatorname{Re}(h)>\frac{1}{2}(p-2),
\end{aligned}
$$

respectively, in (2.3) and integrating $A$, the moment expression is rewritten as

$$
\begin{aligned}
E_{\gamma}\left(U^{h}\right)= & \frac{2^{(1 / 2) n p} K_{p}\left(n, \Sigma_{0}, v\right) \Gamma_{p}((1 / 2) n+h)}{\Gamma(h) \Gamma_{p-1}(h)} \\
& \times \int_{0}^{\infty} y_{1}^{h-1} \int_{Y_{22}>0} \operatorname{det}\left(Y_{22}\right)^{h-(1 / 2) p} \operatorname{det}\left(\Sigma_{0}^{-1}+Y\right)^{-(1 / 2) n-h} \\
& \times{ }_{1} F_{1}^{(p)}\left(\frac{1}{2} n+h ; \frac{1}{2} n ; \frac{1}{2} c_{\gamma}^{2} \Sigma_{0}^{-1}\left(\Sigma_{0}^{-1}+Y\right)^{-1} \Sigma_{0}^{-1} \boldsymbol{v} \boldsymbol{v}^{\prime}\right) d y_{1} d Y_{22},
\end{aligned}
$$

where $Y=\left(\begin{array}{cc}y_{1} & 0 \\ 0 & Y_{22}\end{array}\right)$ and ${ }_{1} F_{1}^{(p)}$ is the confluent hypergeometric function of matrix argument (Gupta and Nagar [4]). Since $\operatorname{rank}\left(\Sigma_{0}^{-1}\left(\Sigma_{0}^{-1}+Y\right)^{-1} \Sigma_{0}^{-1} \boldsymbol{v} \boldsymbol{v}^{\prime}\right)=1$, the only nonzero characteristic root of the matrix $\Sigma_{0}^{-1}\left(\Sigma_{0}^{-1}+Y\right)^{-1} \Sigma_{0}^{-1} \boldsymbol{v} \boldsymbol{v}^{\prime}$ is $\operatorname{tr}\left(\left(\Sigma_{0}^{-1}+Y\right)^{-1} \Sigma_{0}^{-1} \boldsymbol{v} \boldsymbol{v}^{\prime} \Sigma_{0}^{-1}\right)$ and therefore,

$$
\begin{aligned}
&{ }_{1} F_{1}^{(p)}(\left.\frac{1}{2} n+h ; \frac{1}{2} n ; \frac{1}{2} c_{\gamma}^{2} \Sigma_{0}^{-1}\left(\Sigma_{0}^{-1}+Y\right)^{-1} \Sigma_{0}^{-1} \boldsymbol{v} \boldsymbol{v}^{\prime}\right) \\
& \quad={ }_{1} F_{1}\left(\frac{1}{2} n+h ; \frac{1}{2} n ; \frac{1}{2} c_{\gamma}^{2} \operatorname{tr}\left(\left(\Sigma_{0}^{-1}+Y\right)^{-1} \Sigma_{0}^{-1} \boldsymbol{v} \boldsymbol{v}^{\prime} \Sigma_{0}^{-1}\right)\right),
\end{aligned}
$$

where ${ }_{1} F_{1}$ is the confluent hypergeometric function of scalar argument (see [6]). Substituting (2.6) in (2.5) and expanding ${ }_{1} F_{1}$ in series form, the moment expression simplifies to

$$
\begin{aligned}
E_{\gamma}\left(U^{h}\right)= & \frac{2^{(1 / 2) n p} K_{p}\left(n, \Sigma_{0}, \boldsymbol{v}\right) \Gamma_{p}((1 / 2) n+h)}{\Gamma(h) \Gamma_{p-1}(h)} \sum_{t=0}^{\infty}\left(\frac{c_{\gamma}^{2}}{2}\right)^{t} \frac{((1 / 2) n+h)_{t}}{((1 / 2) n)_{t} t !} \\
& \times \int_{0}^{\infty} y_{1}^{h-1} \int_{Y_{22}>0} \operatorname{det}\left(Y_{22}\right)^{h-(1 / 2) p} \operatorname{det}\left(\Sigma_{0}^{-1}+Y\right)^{-(1 / 2) n-h} \\
& \times\left[\boldsymbol{v}^{\prime} \Sigma_{0}^{-1}\left(\Sigma_{0}^{-1}+Y\right)^{-1} \Sigma_{0}^{-1} \boldsymbol{v}\right]^{t} d y_{1} d Y_{22},
\end{aligned}
$$


where $(a)_{r}=a(a+1) \cdots(a+r-1)$ and $(a)_{0}=1$. Noting that $\Sigma_{0}$ is a block diagonal matrix, we obtain

$$
\begin{gathered}
{\left[\boldsymbol{v}^{\prime} \Sigma_{0}^{-1}\left(\Sigma_{0}^{-1}+Y\right)^{-1} \Sigma_{0}^{-1} \boldsymbol{v}\right]^{t}} \\
=\left[v_{1}^{2} \sigma_{11}^{-1}\left(1+\sigma_{11} y_{1}\right)^{-1}+\boldsymbol{v}_{2}^{\prime} \Sigma_{22}^{-1}\left(\Sigma_{22}^{-1}+Y_{2}\right)^{-1} \Sigma_{22}^{-1} \boldsymbol{v}_{2}\right]^{t} \\
=\sum_{k+\ell=t} \frac{t !}{k ! \ell !}\left\{v_{1}^{2} \sigma_{11}^{-1}\left(1+\sigma_{11} y_{1}\right)^{-1}\right\}^{k}\left\{\boldsymbol{v}_{2}^{\prime} \Sigma_{22}^{-1}\left(\Sigma_{22}^{-1}+Y_{22}\right)^{-1} \Sigma_{22}^{-1} \boldsymbol{v}_{2}\right\}^{\ell} \\
\operatorname{det}\left(\Sigma_{0}^{-1}+Y\right)=\left(\sigma_{11}\right)^{-1}\left(1+\sigma_{11} y_{1}\right) \operatorname{det}\left(\Sigma_{22}\right)^{-1} \operatorname{det}\left(I_{p-1}+\Sigma_{22} Y_{22}\right)
\end{gathered}
$$

Now substituting (2.8) in (2.7), we have

$$
\begin{aligned}
E_{\gamma}\left(U^{h}\right)= & \frac{2^{(1 / 2) n p} K_{p}\left(n, \Sigma_{0}, \boldsymbol{v}\right) \Gamma_{p}((1 / 2) n+h)}{\operatorname{det}\left(\Sigma_{0}\right)^{(1 / 2) n+h} \Gamma(h) \Gamma_{p-1}(h)} \\
& \times \sum_{t=0}^{\infty}\left(\frac{c_{\gamma}^{2}}{2}\right)^{t} \frac{((1 / 2) n+h)_{t}}{((1 / 2) n)_{t}} \sum_{k+\ell=t} \frac{1}{k ! \ell !}\left(\frac{v_{1}^{2}}{\sigma_{11}}\right)^{k} \\
& \times \int_{0}^{\infty} y_{1}^{h-1}\left(1+\sigma_{11} y_{1}\right)^{-((1 / 2) n+h+k)} d y_{1} \\
& \times \int_{Y_{22}>0} \operatorname{det}\left(Y_{22}\right)^{h-(1 / 2) p} \operatorname{det}\left(I_{p-1}+\Sigma_{22} Y_{22}\right)^{-(1 / 2) n-h} \\
& \times\left\{\boldsymbol{v}_{2}^{\prime} \Sigma_{22}^{-1}\left(\Sigma_{22}^{-1}+Y_{22}\right)^{-1} \Sigma_{22}^{-1} \boldsymbol{v}_{2}\right\}^{\ell} d Y_{22} .
\end{aligned}
$$

Substituting $Z=\left(I_{p-1}+\Sigma_{22}^{1 / 2} Y_{22} \Sigma_{22}^{1 / 2}\right)^{-1}$, the integral involving $Y_{22}$ is evaluated as

$$
\begin{aligned}
\int_{Y_{22}>0} & \operatorname{det}\left(Y_{22}\right)^{h-(1 / 2) p} \operatorname{det}\left(I_{p-1}+\Sigma_{22} Y_{22}\right)^{-(1 / 2) n-h}\left\{\boldsymbol{v}_{2}^{\prime} \Sigma_{22}^{-1}\left(\Sigma_{22}^{-1}+Y_{22}\right)^{-1} \Sigma_{22}^{-1} \boldsymbol{v}_{2}\right\}^{\ell} d Y_{22} \\
= & \operatorname{det}\left(\Sigma_{22}\right)^{-h} \int_{0<Z<I_{p-1}} \operatorname{det}(Z)^{(1 / 2)(n-p)} \\
& \times \operatorname{det}\left(I_{p-1}-Z\right)^{h-(1 / 2) p}\left(\boldsymbol{v}_{2}^{\prime} \Sigma_{22}^{-1 / 2} Z \Sigma_{22}^{-1 / 2} \boldsymbol{v}_{2}\right)^{\ell} d Z \\
= & \operatorname{det}\left(\Sigma_{22}\right)^{-h}\left(\frac{\partial}{\partial \eta}\right)_{\eta=0}^{\ell} \int_{0<Z<I_{p-1}} \operatorname{det}(Z)^{(1 / 2)(n-p)} \operatorname{det}\left(I_{p-1}-Z\right)^{h-(1 / 2) p} \\
& \times \operatorname{etr}\left(\eta \boldsymbol{v}_{2}^{\prime} \Sigma_{22}^{-1 / 2} Z \Sigma_{22}^{-1 / 2} \boldsymbol{v}_{2}\right) d Z \\
= & \operatorname{det}\left(\Sigma_{22}\right)^{-h} \frac{\Gamma_{p-1}((1 / 2) n) \Gamma_{p-1}(h)}{\Gamma_{p-1}((1 / 2) n+h)}\left(\frac{\partial}{\partial \eta}\right)_{\eta=0}^{\ell}{ }_{1}^{(p-1)}\left(\frac{1}{2} n ; \frac{1}{2} n+h ; \eta \Sigma_{22}^{-1} \boldsymbol{v}_{2} \boldsymbol{v}_{2}^{\prime}\right) \\
= & \operatorname{det}\left(\Sigma_{22}\right)^{-h} \frac{\Gamma_{p-1}((1 / 2) n) \Gamma_{p-1}(h)}{\Gamma_{p-1}((1 / 2) n+h)} \frac{((1 / 2) n)_{\ell}}{((1 / 2) n+h)_{\ell}}\left(\boldsymbol{v}_{2}^{\prime} \Sigma_{22}^{-1} \boldsymbol{v}_{2}\right)^{\ell},
\end{aligned}
$$

where ${ }_{1} F_{1}^{(p-1)}$ is the confluent hypergeometric function of matrix argument (see [4]). 
Collecting terms containing $y_{1}$ and integrating, we obtain

$$
\int_{0}^{\infty} y_{1}^{h-1}\left(1+\sigma_{11} y_{1}\right)^{-((1 / 2) n+h+k)} d y_{1}=\sigma_{11}^{-h} \frac{\Gamma((1 / 2) n) \Gamma(h)}{\Gamma((1 / 2) n+h)} \frac{((1 / 2) n)_{k}}{((1 / 2) n+h)_{k}} .
$$

Substituting (2.10), (2.11), and (1.7) in (2.9) and simplifying the resulting expression using results on gamma function, we get

$$
\begin{aligned}
E_{\gamma}\left(U^{h}\right)= & \exp \left(-\frac{1}{2} c_{\gamma}^{2} \boldsymbol{v}^{\prime} \Sigma_{0}^{-1} \boldsymbol{v}\right) \frac{\Gamma((1 / 2) n)}{\Gamma[(1 / 2)(n-p+1)]} \sum_{t=0}^{\infty} \sum_{\ell+k=t}\left(\frac{c_{\gamma}^{2}}{2}\right)^{t} \frac{((1 / 2) n)_{k}((1 / 2) n)_{\ell}}{((1 / 2) n)_{t}} \\
& \times \frac{\left(v_{1}^{2} / \sigma_{11}\right)^{k}\left(\boldsymbol{v}_{2}^{\prime} \Sigma_{22}^{-1} \boldsymbol{v}_{2}\right)^{\ell}}{k ! \ell !} \frac{\Gamma[(1 / 2)(n-p+1)+h] \Gamma((1 / 2) n+t+h)}{\Gamma((1 / 2) n+k+h) \Gamma((1 / 2) n+\ell+h)} \\
= & \exp \left(-\frac{1}{2} c_{\gamma}^{2} \boldsymbol{v}^{\prime} \Sigma_{0}^{-1} \boldsymbol{v}\right) \frac{\Gamma((1 / 2) n) \Gamma[(1 / 2)(n-p+1)+h]}{\Gamma((1 / 2) n+h) \Gamma[(1 / 2)(n-p+1)]} \sum_{k=0}^{\infty}\left(\frac{c_{\gamma}^{2}}{2}\right)^{k} \\
& \times \frac{\left(v_{1}^{2} / \sigma_{11}\right)^{k}}{k !}{ }_{2} F_{2}\left(\frac{1}{2} n+h+k, \frac{1}{2} n ; \frac{1}{2} n+k, \frac{1}{2} n+h ; \frac{1}{2} c_{\gamma}^{2} \boldsymbol{v}_{2}^{\prime} \Sigma_{22}^{-1} \boldsymbol{v}_{2}\right),
\end{aligned}
$$

where ${ }_{2} F_{2}$ is the generalized hypergeometric function of scalar argument (see [6]).

3. Distribution of $R^{2}$ under mixture normal model. The density function $f(u)$ of $U=1-R^{2}$ is obtained by taking the inverse Mellin transform of $E\left(U^{h}\right)$ as

$$
f(u)=\sum_{\gamma=0}^{N}\left(\begin{array}{l}
N \\
\gamma
\end{array}\right) \epsilon^{\gamma}(1-\epsilon)^{N-\gamma} f_{\gamma}(u)
$$

with

$$
f_{\gamma}(u)=(2 \pi \iota)^{-1} \int_{C} E_{\gamma}\left(U^{h}\right) u^{-h-1} d h, \quad 0<u<1,
$$

where $\iota=\sqrt{-1}$ and $C$ is a suitable contour. Substituting (2.12) in (3.2), we obtain

$$
\begin{aligned}
f_{\gamma}(u)= & \exp \left(-\frac{1}{2} c_{\gamma}^{2} \boldsymbol{v}^{\prime} \Sigma_{0}^{-1} \boldsymbol{v}\right) \frac{\Gamma((1 / 2) n)}{\Gamma[(1 / 2)(p-1)] \Gamma[(1 / 2)(n-p+1)]} \sum_{t=0}^{\infty} \sum_{k+\ell=t}\left(\frac{c_{\gamma}^{2}}{2}\right)^{t} \\
& \times \frac{((1 / 2) n)_{k}((1 / 2) n)_{\ell}}{((1 / 2) n)_{t}} \frac{\left(v_{1}^{2} / \sigma_{11}\right)^{\ell}\left(v_{2}^{\prime} \Sigma_{22}^{-1} \boldsymbol{v}_{2}\right)^{k}}{k ! \ell !} u^{(1 / 2) n+k+\ell-1}(1-u)^{(1 / 2)(p-3)} \\
& \times{ }_{2} F_{1}\left(\frac{1}{2}(p-1)+k, \frac{1}{2}(p-1)+\ell ; \frac{1}{2}(p-1) ; 1-u\right), \quad 0<u<1,
\end{aligned}
$$

where ${ }_{2} F_{1}$ is the Gauss hypergeometric function (see [6]). To obtain (3.3) we have used the result

$$
\begin{gathered}
\int_{0}^{1} u^{(1 / 2) n+h+k+\ell-1}(1-u)^{(1 / 2)(p-3)}{ }_{2} F_{1}\left(\frac{1}{2}(p-1)+k, \frac{1}{2}(p-1)+\ell ; \frac{1}{2}(p-1) ; 1-u\right) d u \\
=\frac{\Gamma[(1 / 2)(p-1)] \Gamma[(1 / 2)(n-p+1)+h] \Gamma((1 / 2) n+t+h)}{\Gamma((1 / 2) n+k+h) \Gamma((1 / 2) n+\ell+h)} .
\end{gathered}
$$


The density of $R^{2}=1-U$ is now derived from the density of $U$ as

$$
g\left(R^{2}\right)=\sum_{\gamma=0}^{N}\left(\begin{array}{l}
N \\
\gamma
\end{array}\right) \epsilon^{\gamma}(1-\epsilon)^{N-\gamma} g_{\gamma}\left(R^{2}\right),
$$

where

$$
\begin{aligned}
g_{\gamma}\left(R^{2}\right)= & \exp \left(-\frac{1}{2} c_{\gamma}^{2} \boldsymbol{v}^{\prime} \Sigma_{0}^{-1} \boldsymbol{v}\right) \frac{\Gamma((1 / 2) n)}{\Gamma[(1 / 2)(p-1)] \Gamma[(1 / 2)(n-p+1)]} \sum_{t=0}^{\infty} \sum_{k+\ell=t}\left(\frac{c_{\gamma}^{2}}{2}\right)^{t} \\
& \times \frac{((1 / 2) n)_{k}((1 / 2) n)_{\ell}}{((1 / 2) n)_{t}} \frac{\left(v_{1}^{2} / \sigma_{11}\right)^{\ell}\left(\boldsymbol{v}_{2}^{\prime} \Sigma_{22}^{-1} \boldsymbol{v}_{2}\right)^{k}}{k ! \ell !} \\
& \times\left(1-R^{2}\right)^{(1 / 2) n+k+\ell-1}\left(R^{2}\right)^{(1 / 2)(p-3)} \\
& \times{ }_{2} F_{1}\left(\frac{1}{2}(p-1)+k, \frac{1}{2}(p-1)+\ell ; \frac{1}{2}(p-1) ; R^{2}\right), \quad 0<R^{2}<1 .
\end{aligned}
$$

By using the result ${ }_{2} F_{1}(a, b ; c ; z)=(1-z)^{c-a-b}{ }_{2} F_{1}(c-a, c-b ; c ; z)$, the above density can be rewritten as

$$
\begin{aligned}
g_{\gamma}\left(R^{2}\right)= & \exp \left(-\frac{1}{2} c_{\gamma}^{2} \boldsymbol{v}^{\prime} \Sigma_{0}^{-1} \boldsymbol{v}\right) \frac{\Gamma((1 / 2) n)}{\Gamma[(1 / 2)(p-1)] \Gamma[(1 / 2)(n-p+1)]} \\
& \times\left(R^{2}\right)^{(1 / 2)(p-3)}\left(1-R^{2}\right)^{(1 / 2)(n-p-1)} \sum_{t=0}^{\infty} \sum_{k+\ell=t}\left(\frac{c_{\gamma}^{2}}{2}\right)^{t} \frac{((1 / 2) n)_{k}((1 / 2) n)_{\ell}}{((1 / 2) n)_{t}} \\
& \times \frac{\left(v_{1}^{2} / \sigma_{11}\right)^{\ell}\left(\boldsymbol{v}_{2}^{\prime} \Sigma_{22}^{-1} \boldsymbol{v}_{2}\right)^{k}}{k ! \ell !}{ }_{2} F_{1}\left(-k,-\ell ; \frac{1}{2}(p-1) ; R^{2}\right), \quad 0<R^{2}<1 .
\end{aligned}
$$

It is interesting to note that if $\boldsymbol{v}=\mathbf{0}$, then the density $g\left(R^{2}\right)$ reduces to

$$
\begin{aligned}
g\left(R^{2}\right)= & \frac{\Gamma((1 / 2) n)}{\Gamma[(1 / 2)(p-1)] \Gamma[(1 / 2)(n-p+1)]} \\
& \times\left(R^{2}\right)^{(1 / 2)(p-3)}\left(1-R^{2}\right)^{(1 / 2)(n-p-1)}, \quad 0<R^{2}<1 .
\end{aligned}
$$

\section{REFERENCES}

[1] A. K. A. Amey, Robustness of the multiple correlation coefficient when sampling from a mixture of two multivariate normal populations, Comm. Statist. Simulation Comput. 19 (1990), no. 4, 1443-1457.

[2] A. K. A. Amey and A. K. Gupta, Testing sphericity under a mixture model, Austral. J. Statist. 34 (1992), 451-460.

[3] A. K. Gupta and D. G. Kabe, On some noncentral distribution problems for the mixture of two normal populations, Metrika 38 (1991), 1-10.

[4] A. K. Gupta and D. K. Nagar, Matrix Variate Distributions, Chapman \& Hall/CRC, Florida, 2000.

[5] D. G. Kabe and A. K. Gupta, Hotelling's $T^{2}$-distribution for a mixture of two normal populations, South African Statist. J. 24 (1990), 87-92. 
[6] Y. L. Luke, The Special Functions and Their Approximations, Vol. I, Academic Press, New York, 1969.

[7] D. K. Nagar and M. E. Castañeda, Distribution of correlation coefficient under mixture normal model, to appear in Metrika, 2002.

[8] M. S. Srivastava, On the distribution of Hotelling's $T^{2}$ and multiple correlation $R^{2}$ when sampling from a mixture of two normals, Comm. Statist. A-Theory Methods 12 (1983), no. 13, 1481-1497.

[9] M. S. Srivastava and H. M. Awan, On the robustness of Hotelling's $T^{2}$-test and distribution of linear and quadratic forms in sampling from a mixture of two multivariate normal populations, Comm. Statist. A-Theory Methods 11 (1982), no. 1, 81-107.

[10] On On the robustness of the correlation coefficient in sampling from a mixture of two bivariate normals, Comm. Statist. A-Theory Methods 13 (1984), 371-382.

[11] W. Y. Tan, On the distribution of the sample covariance matrix from a mixture of normal densities, South African Statist. J. 12 (1978), 47-55.

[12] D. M. Titterington, A. F. M. Smith, and U. E. Makov, Statistical Analysis of Finite Mixture Distributions, John Wiley, Chichester, 1985.

HydAR Ali: DePartment of MATHEMATics AND COMPUTER SCIENCE, THE UNIVERSiTy OF THE WeST INDIES, ST. AUguSTINE, TRINIDAD AND TOBAGO

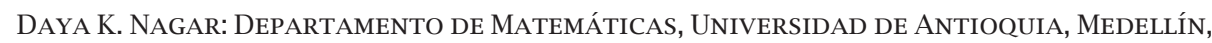
A. A. 1226, COLOMBIA 


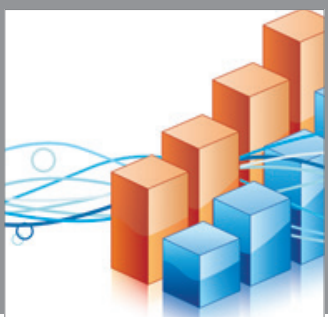

Advances in

Operations Research

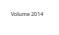

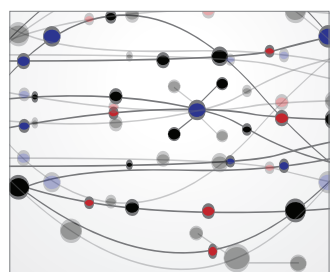

\section{The Scientific} World Journal
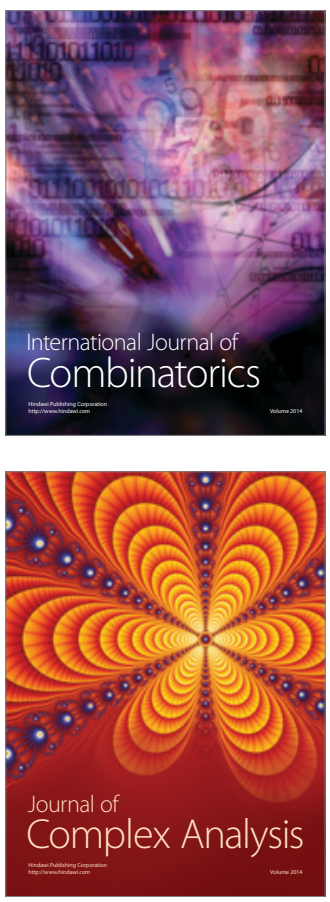

International Journal of

Mathematics and

Mathematical

Sciences
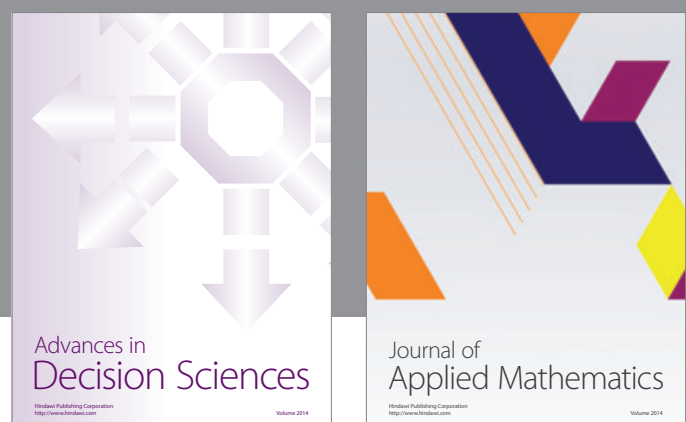

Journal of

Applied Mathematics
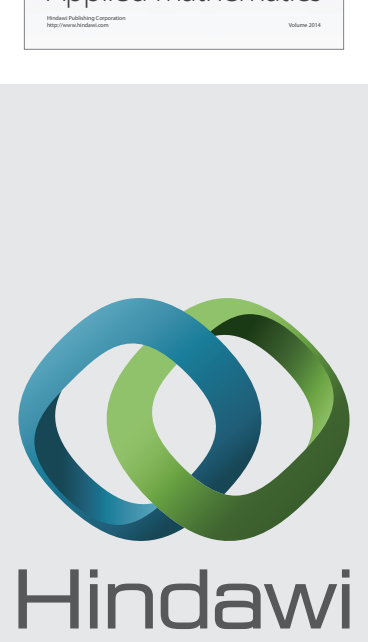

Submit your manuscripts at http://www.hindawi.com
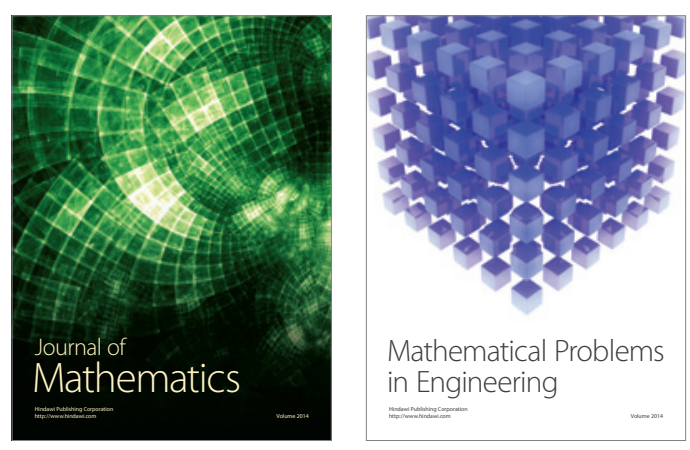

Mathematical Problems in Engineering
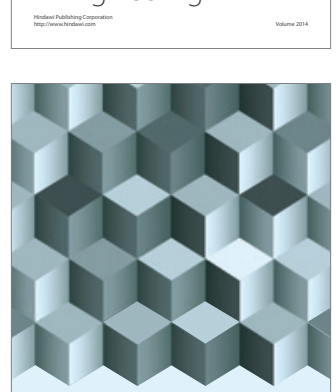

Journal of

Function Spaces
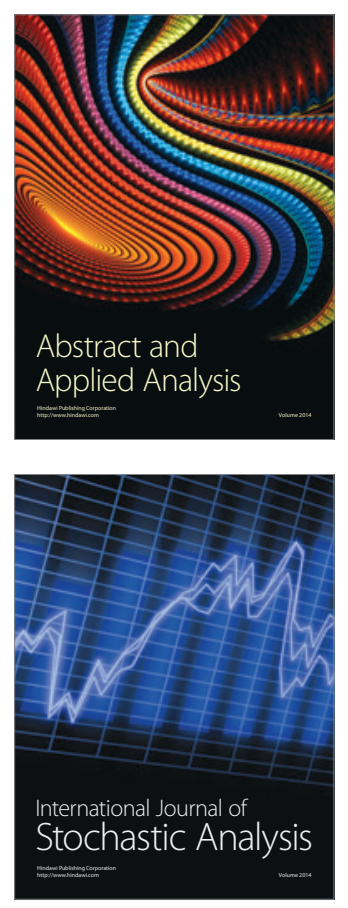

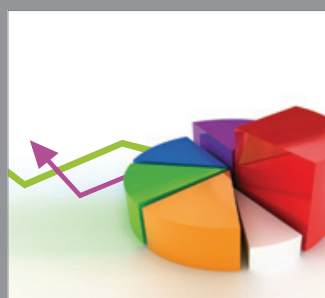

ournal of

Probability and Statistics

Promensencen
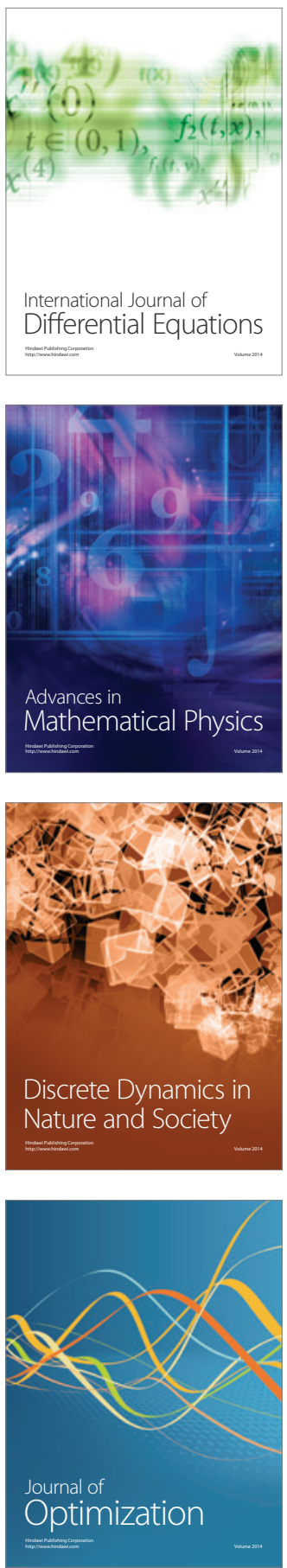